\title{
Wave Operators for Classical Particle Scattering
}

\author{
BARRY SIMON \\ Departments of Mathematics and Physics, Princeton University \\ Princeton, New Jersey, USA
}

Received June 1, 1971

\begin{abstract}
We discuss the fundamentals of classical particle scattering of a two body system in forces which are $0\left(r^{-2-\varepsilon}\right)$ at infinity along with their Lipshitz constants. We prove asymptotic completeness for this two-body case. Of particular interest is the fact that in the absence of control on Lipshitz constants at $\infty$, two solutions of the interacting equation may be asymptotic to the same free solution at $-\infty$.
\end{abstract}

\section{§ 1. Introduction}

During the past fifteen years, one of the most studied areas of mathematical physics has been the rigorous foundations of scattering theory. There have been studies of the quantum scattering of two bodies in short range [1] and long range forces [2], of $n$-body quantum systems [3], of potential scattering with free dynamics given by relativistic wave equations [4], of accoustical scattering [5], of scattering in the general theory of quantum field [6], of scattering of quantized particles in the presence of unquantized external electromagnetic fields [7], of scattering in model field theories [8] and of classical field theoretic scattering [9]. Suprisingly there has been very little study of the most classical of scattering problems: the scattering of classical particles moving in Newtonian force fields. Hunziker [10] and Cook [11] have briefly studied classical scattering by taking $L^{2}$ of phase space and using the Hilbert space techniques developed for quantum particle scattering $[1,3]$. In this note, we wish to study classical particle scattering directly in phase space; this approach is in many ways more natural than Hunziker's construction involving $L^{2}$ of phase space. As we will discuss in $\S 7$, up to sets of measure zero, Hunziker's results imply most of ours but the two methods of proof are very different.

The basic existence theorem we will prove is that given a solution of the free equation $\vec{r}_{o}(t)=\vec{a}+\vec{b} t$, there exists a solution, $\vec{r}(t)$, of the interacting equation so that $\lim _{t \rightarrow-\infty}\left|\vec{r}(t)-\vec{r}_{o}(t)\right|+\left|\dot{\vec{r}}(t)-\dot{\vec{r}}_{o}(t)\right|=0$. We view this as an existence theorem for solutions with boundary conditions at

Research partially supported by U.S. Air Force under contract AF49(638) 1545. 
$t=-\infty$. There is an interesting analogy between this theory developed in $\S 3$ and the standard existence and uniqueness theorems for ordinary differential equations (O.D.E.) with initial conditions at some finite time [12]. Both theories depend on using fixed point theorems and as in the O.D.E. case we will only have existence and uniqueness when we have control over the falloff of the forces and Lipshitz constants at infinity. When we only have control on the forces, we will be able to prove existence but we will present examples to show uniqueness does not hold.

Once we have existence and uniqueness of solutions of the interacting equation asymptotic to $\vec{a}+\vec{b} t$ there is a natural map $\Omega^{+}: R^{6} \rightarrow R^{6}$ given by $\Omega^{+}(\vec{a}, \vec{b})=(\vec{r}(0), \dot{\vec{r}}(0))$. Similarly, a map $\Omega^{-}$is defined for solutions asymptotic to a given free solution at $t=+\infty . \Omega^{+}$is analogous to the wave operator of quantum scattering $[13,1]$. In both cases $\Omega^{+} p$ is that $t=0$ interacting data which at $t=-\infty$, is asymptotic to the free solution with data, $p$, at $t=0$. In quantum mechanical scattering, $\Omega^{+}$is an isometry and the analog of this property in classical scattering is that $\Omega^{+}$is measure preserving, a fact proven in $\S 4$. Since it is known that except for a set of interacting data measure $0, \lim _{t \rightarrow-\infty}|r(t)|=\infty$ if and only if $\lim _{t \rightarrow+\infty}|r(t)|=\infty$ [14], (see also [10]) we will be able to prove (in $\S 5$ ) that $\operatorname{Ran} \Omega^{+}=\operatorname{Ran} \Omega^{-}$up to sets of measure 0 . The $S$-matrix $\left(\Omega^{-}\right)^{-1} \Omega^{+}$will then be defined as a bijection on $R^{6}$ (up to sets of measure zero).

Let us consider how this picture differs from the more usual picture of classical central two-body scattering [15] in terms of scattering angle as a function of impact parameter. In the central case, $S: R^{6} \rightarrow R^{6}$ will be rotationally invariant; thus $S:(\vec{a}, \vec{b}) \rightarrow\left(\vec{a}^{\prime}, \vec{b}^{\prime}\right)$ is determined by knowing $S(\vec{a}, p \hat{e})$ where $\hat{e}$ is some fixed reference direction. By conservation of energy, $\left|\vec{b}^{\prime}\right|=p$. Moreover, since $S$ commutes with the free dynamics we may assume $\vec{a}$ is orthogonal to $\hat{e}$. By conservation of angular momentum, the plane of $\vec{a}^{\prime}$ is known once $\vec{b}^{\prime}$ is known as is the component of $\vec{a}^{\prime}$ orthogonal to $\vec{b}^{\prime}$. If $(m \equiv 1) T=(-1)$ component of $\vec{a}^{\prime}$ parallel to $\vec{b}^{\prime} /$ length of $\vec{b}^{\prime}$, we conclude $S$ is given by two functions, the cosine of the scattering angle $\hat{e} \cdot \hat{e}^{\prime}$ as a function of $p$ and $|\vec{a} \times \vec{b}| /|b|$ and the time delay, $T$, as a function of the same scalar variables. In the usual picture of central scattering, one throws this extra information of time delay away (we give an explicit formula for it in Appendix 3).

Except for some remarks on $n$-body scattering in $\S 6$, we will consider a two body system in the center of mass, so the equation of motion will be

$$
\frac{d^{2} \vec{r}}{d t^{2}}=\vec{F}(\vec{r}(t))
$$

where $\vec{F}$ is a given force. 
We will make some subset of the following assumptions at all times:

(A) $\vec{F}$ is a Lipshitz function with $\sup _{\vec{r}}|\vec{F}(\vec{r})|<\infty$.

(B) For some $R_{0}, \alpha>2$ and $A$,

$$
|\vec{F}(\vec{r})| \leqq A r^{-\alpha} \quad \text { if } \quad r \geqq R_{0} .
$$

(C) For some $R_{1}, \beta>2$ and $B$,

$$
|\vec{F}(\vec{x})-\vec{F}(y)| \leqq B r^{-\beta}|\vec{x}-\vec{y}| \quad \text { if } \quad|\vec{x}|,|\vec{y}| \geqq r \geqq R_{1}
$$

(D) $\vec{F}$ is conservative, i.e. $\vec{F}=-\vec{\nabla} V$ with $V \rightarrow 0$ at $r=\infty$.

We will always suppose (A) in order to have global existance and uniqueness of solutions of Newton's equations. We could weaken (A) to allow some sorts of finite singularities in $\vec{F}$. (B) will imply existance of $\Omega^{+}$but not uniqueness (see Appendix 1) and (C) will imply uniqueness. (B) breaks down at the Coulomb force where it is known solutions aren't asymptotic to free solutions. Appendix 3 shows (C) is a best possible condition for uniqueness. (D) will be useful for $\S 4$ and $\S 5$ but we expect it is not essential for $\S 5$.

\section{§ 2. The Integral Equation for Scattering}

Throughout this section, we will suppose condition (A) on the forces holds. We are seeking solutions of $\ddot{\vec{r}}(t)=\vec{F}(\vec{r}(t))$ where $\vec{r}(t)=\vec{a}+\vec{b} t+\vec{u}(t)$ where $\vec{b} \neq 0$ and $\lim _{t \rightarrow-\infty}|\vec{u}(t)|+|\dot{\vec{u}}(t)|=0$. We can then define the wave operator as $\Omega^{+}(\vec{a}, \vec{b})=(\vec{r}(0), \dot{\vec{r}}(0))$. To find a solution of the interacting equation with the right behavior at $t=-\infty$, we use the usual trick of rewriting a differential equation with boundary condition as an integral equation:

Lemma 1. Let $F$ obey (A). Suppose $\vec{u}(t)$ is continuous and satisfies

$$
\vec{u}(t)=\int_{-\infty}^{t} d s \int_{-\infty}^{s} d \tau \vec{F}(\vec{a}+\vec{b} \tau+\vec{u}(\tau))
$$

(in the sense that the integral is absolutely convergent). Then $u$ satisfies

$$
\ddot{\vec{r}}(t)=\vec{F}(\vec{r}(t))
$$

with $\vec{r}(t)=\vec{u}(t)+\vec{a}+\vec{b}$. Moreover $\lim _{t \rightarrow-\infty}|\vec{u}(t)|+|\dot{\vec{u}}(t)|=0$.

Proof. Since the forces are continuous and the integral is assumed absolutely convergent, the result is simple. 
The useful thing about condition (B) is that a strong "converse" of Lemma 1 holds:

Lemma 2. Suppose $\vec{r}(t)$ satisfies $\ddot{\vec{r}}(t)=\vec{F}(\vec{r}(t))$ where $F$ satisfies (A) and (B) and that $|\vec{r}(t)| \geqq C t$ for some $C>0$ and all $t>T_{0}$ for some $T_{0}$. Then

(a) $\lim _{t \rightarrow \infty} \overrightarrow{\vec{r}}(t)=\vec{b}$ and $\lim _{t \rightarrow+\infty} \vec{r}(t)-\vec{b} t=\vec{a}$ exists

(b) If $\vec{u}(t)=\vec{r}(t)-\vec{a}-\vec{b} t$, then $\vec{u}$ obeys:

$$
\vec{u}(t)=\int_{t}^{\infty} d s \int_{s}^{\infty} d \tau \vec{F}(\vec{u}(\tau)+\vec{a}+\vec{b} \tau)
$$

where the integral is absolutely convergent.

Remark. We have written lemmas 1 and 2 unsymmetrically in $t \rightarrow-\infty$ and $t \rightarrow+\infty$, because we tend to think of studying $\Omega^{+}$and $\left(\Omega^{-}\right)^{-1}$.

Proof. Since $|\vec{r}(t)| \geqq C t, \quad|\vec{F}(\vec{r}(t))| \leqq A C^{-\alpha} t^{-\alpha}$ for $t>T_{1}$ where $T_{1}=\max \left(T_{0}, R_{0} / C\right)$. Since $\alpha>2$,

As a result:

$$
\int_{T_{1}}^{\infty} d t|\vec{F}(\vec{r}(t))|<\infty .
$$

$$
\vec{b}=\dot{\vec{r}}\left(T_{1}\right)+\int_{T_{1}}^{\infty} d t \vec{F}(\vec{r}(t))=\lim _{t \rightarrow \infty}\left[\dot{\vec{r}}\left(T_{1}\right)+\int_{T_{1}}^{t} d t \vec{F}(\vec{r}(t))\right]=\lim _{t \rightarrow \infty} \dot{\vec{r}}(t)
$$

exists. Moreover, for any $s>T_{1}$,

$$
\vec{b}=\dot{\vec{r}}(s)+\int_{s}^{\infty} d t \vec{F}(\vec{r}(t))
$$

Since $|\vec{F}(\vec{r}(t))| \leqq A C^{-\alpha} t^{-\alpha}$ and $\alpha>2$,

so

$$
\left|\int_{s}^{\infty} d t \vec{F}(\vec{r}(t))\right| \leqq A(\alpha-1)^{-1} C^{-\alpha} S^{-\alpha+1}
$$

$$
\begin{aligned}
\vec{a}=\vec{r}\left(T_{1}\right) & =\vec{b} T_{1}-\int_{T_{1}}^{\infty} d s \int_{s}^{\infty} d t \vec{F}(\vec{r}(t))=\lim _{t \rightarrow \infty} \vec{r}\left(T_{1}\right)-\vec{b} T_{1}-\int_{T_{1}}^{T} d s(\vec{b}-\dot{\vec{r}}(s)) \\
& =\lim _{t \rightarrow \infty} \vec{r}(t)-\vec{b} t
\end{aligned}
$$

exists. For any $T>T_{1}$,

$$
\vec{a}=\vec{r}(T)-\vec{b} T-\int_{T}^{\infty} d s \int_{S}^{\infty} d t \vec{F}(\vec{r}(t))
$$

so $u$ given by $\vec{u}(T)=\vec{r}(T)-\vec{a}-\vec{b} T$ satisfies the integral equation. 


\section{§ 3. Existence of the Wave Operators}

Throughout this section we will suppose (A) and (B) hold. Fix $\vec{a}$ and $\vec{b} \neq 0$. For $\vec{u}(t)$ a continuous function on $(-\infty, T)$ with $\|u\|_{\infty}<1$, we have $|\vec{r}(t)|>C|t|$ for $t<T_{0}$ where $T_{0}$ and $C$ are choosen suitably. Thus

exists. We thus define

$$
\int_{-\infty}^{t} d s \int_{-\infty}^{s} d \tau \vec{F}(\vec{a}+\vec{b} \tau+\vec{u}(\tau))
$$

$$
(\mathscr{F} u)(t)=\int_{-\infty}^{t} d s \int_{-\infty}^{s} d \tau \vec{F}(\vec{a}+\vec{b} \tau+\vec{u}(\tau))
$$

We are seeking fixed points of $\mathscr{F}$. We first note:

Lemma 3. Let $\vec{a}, \vec{b} \neq 0$ be given. Suppose $\vec{F}$ obeys conditions (A) and (B). Then there exists $T$ so that whenever $\vec{u} \varepsilon\{\vec{u} \mid \vec{u}$ is continuous on $(-\infty, T)$; $\left.\|u\|_{\infty} \leqq 1\right\}, \equiv C_{T}$ then $\mathscr{F} u \varepsilon C_{T}$. In fact, there exists $C_{1}$ and $C_{2}$ so that

$$
\overrightarrow{\mathscr{F} u} \in\left\{\vec{v}|| \vec{v}(t)-\vec{v}(s)\left|\leqq C_{1}\right| t-\left.s|;| \vec{v}(t)\left|\leqq C_{2}\right| t\right|^{-\alpha+2} ; t \in(-\infty, T)\right\} \equiv \mathscr{E} .
$$

Proof. By taking $T$ negative enough, we can be sure that if $u \in C_{T}$, then $\vec{r}(t)=\vec{a}+\vec{b} t+\vec{u}(\vec{t})$ obeys $|\vec{r}(t)|>C t ;|\vec{r}(t)|>R_{0}$ if $t<T$. Then using condition (B)

$$
|(\overrightarrow{\mathscr{F}} u)(t)| \leqq C^{-\alpha} A(\alpha-1)(\alpha-2)|t|^{-\alpha+2} .
$$

By taking $|T|$ large enough, we can conclude, $|(\mathscr{F} u)(t)| \leqq 1$ if $t<T$. Taking $C_{2}=C^{-\alpha} A(\alpha-1)(\alpha-2)$ and $C_{1}=C^{-\alpha} A(\alpha-1)|T|^{-\alpha+1}$ we have $\mathscr{F} u \in \varepsilon$. The equicontinuity condition $|\vec{v}(t)-\vec{v}(s)| \leqq C_{1}|t-s|$ follows from

$$
\left|(\overrightarrow{\mathscr{F} u})^{\prime}(t)\right| \leqq \int_{-\infty}^{t} d s \mid \vec{F}\left(\vec{a}+\vec{b} s+\left.\vec{u}(s)\left|\leqq C^{-\alpha} A(\alpha-1)\right| t\right|^{-\alpha+1} .\right.
$$

The last part of Lemma 3 allows us to prove:

Theorem 1. If $\vec{F}$ obeys conditions (A) and (B), then for any $\vec{a}, \vec{b} \neq 0$, there is a function $\vec{r}(t)$ solving

$$
\vec{F}(\vec{r}(t))=\ddot{\vec{r}}(t)
$$

with $\lim _{t \rightarrow-\infty}|\vec{r}(t)-\vec{a}-\vec{b} t|+|\dot{r}(t)-\vec{b}|=0$.

Proof. It is enough to find such a function $r$ on $(-\infty, T)$ for some $T$ and then apply the ordinary global existence and uniqueness theorem to extend $r$ to $(-\infty, \infty)$. By Lemma 1 , we need only show $\mathscr{F}$ has a fixed point in some set, $C_{T}$. By Lemma 3 , by taking $T$ sufficiently small, we can be sure that $\mathscr{F}$ takes $C_{T} \cap \mathscr{E}$ into itself. But $C_{T} \cap \mathscr{E}$ is convex and is 
compact in the \|\|$_{\infty}$-topology since $C_{T}$ is closed and $\mathscr{E}$ is compact by equicontinuity arguments. Moreover, it is non-empty. Thus, by the Leray-Schauder-Tychonoff theorem ([16] pp. 453-56) $\mathscr{F}$ has a fixed point in $C_{T} \cap \mathscr{E}$. This proves the existence of a solution.

As the examples in Appendix 1 show, uniqueness of the fixed point may not hold if only (A) and (B) are true but if (C) also holds the situation is very different:

Theorem 2. If $\vec{F}$ obeys (A), (B) and (C), then for any $\vec{a}, \vec{b} \neq 0$, there exists a unique function, $\vec{r}(t)$, obeying the conclusions of Theorem 1 .

Proof. By the global existance and uniqueness theorem, we need only prove existence and uniqueness of fixed points of $\mathscr{F}$ on some $C_{T}$. But by (C), if $u, v \in C_{T}$ with $T$ sufficiently negative

$$
|\mathscr{F} u(t)-\mathscr{F} v(t)| \leqq C^{-\beta} B|t|^{-\beta+2}(\beta-1)(\beta-2)\|u-v\|_{\infty}
$$

so by taking $T$ negative enough, we can be sure that $\mathscr{F}$ is a contraction on $C_{T}$. It thus follows from the contraction mapping theorem that $\mathscr{F}$ has a unique fixed point.

It is known if $F_{a}$ is a family of contractions on the unit ball in a Banach space depending sufficiently smoothly on a real parameter $a$, then the fixed point of $F_{a}$ is a smooth function of $a$. As an example of how this theorem (see [17], pp. 229-234 for an exact statement) may be applied we note:

Theorem 3. If $\vec{F}$ is $C^{\infty}$ with

$$
\lim _{\vec{r} \rightarrow \infty}\left|r^{\alpha+n} \frac{\partial^{n_{1}+n_{2}+n_{3}} F}{\partial x^{n_{1}} \partial y^{n_{2}} \partial z^{n_{3}}}\right|=0
$$

for some $\alpha>2$ (with $n=n_{1}+n_{2}+n_{3}$ ), then the wave operators, $\Omega^{ \pm}$given by Theorem 2 are $C^{\infty}$ functions of $\vec{a}$ and $\vec{b}$.

\section{§ 4. Isometric Nature of $\boldsymbol{\Omega}^{ \pm}$}

To prove $\Omega^{ \pm}$are measure preserving, we introduce the maps $\Omega_{t}$ defined as follows. Let $S_{t}: R^{6} \rightarrow R^{6}$ be the map given by $r(t)=S_{t}\left(q_{o}, p_{o}\right)$ solves $\ddot{\vec{r}}(t)=\vec{F}(\vec{r}(t)) ; r(0)=q_{o} ; \dot{r}(0)=p_{o}$. Let $S_{t}^{o}\left(q_{o}, p_{o}\right)=q_{o}+p_{o} t$. Define

Not surprisingly:

$$
\Omega_{t}=S_{-t} S_{t}^{(o)} .
$$

Lemma 4. Let $\vec{F}$ obey (A), (B), (C). Let $\Omega^{t}$ be defined as above. Then as $t \rightarrow-\infty$

$$
\Omega^{t} \rightarrow \Omega^{+}
$$

uniformly on compacts. 
Proof. Given a compact, $D$, in phase space, we can find a $T$ so that the maps $\mathscr{F}_{a, b}$ given by

$$
\left(\mathscr{F}_{a, b} u\right)(\tau)=\int_{-\infty}^{t} d s \int_{-\infty}^{s} d \tau \vec{F}(\vec{a}+\vec{b} \tau+u(\tau))
$$

are contractions on the set $C_{T}$ introduced in $\S 3$. Let

$$
\left(\mathscr{F}_{a, b}^{(v)} u\right)(s)=\int_{v}^{t} d s \int_{v}^{s} d \tau \vec{F}(\vec{a}+\vec{b} \tau+u(\tau)) .
$$

The $\mathscr{F}_{a, b}^{(v)}$ are also contractions on $C_{T}$ and $\mathscr{F}_{a, b}^{(v)} \rightarrow \mathscr{F}_{a, b}$ uniformly on $C_{T} \times D$. It thus follows that the fixed points $u_{a, b}^{(b)}$ converge in \|\|$_{\infty}$ to the fixed points $u_{a, b}$ of $\mathscr{F}_{a, b}$. Since

$$
\begin{aligned}
\Omega^{t}(a, b) & =S_{T-1}\left(u_{a, b}^{(t)}(T-1), \dot{u}_{a, b}^{(t)}(T-1)\right), \\
\Omega^{+}(a, b) & =S_{T-1}\left(u_{a, b}(T-1), \dot{u}_{a, b}(T-1)\right)
\end{aligned}
$$

we conclude the lemma.

By Liouville theorem $S_{-t}$ and $S_{t}^{(o)}$ are measure preserving so $\Omega^{ \pm}$ are measure preserving. It follows from Lemma 4 that:

Theorem 4. Let $F$ obey (A)-(D). Then $\Omega^{ \pm}$are measure preserving.

Proof. It is enough to prove

$$
\int d \vec{a} d \vec{b} f\left(\Omega^{ \pm}(\vec{a}, \vec{b})\right)=\int d \vec{a} d \vec{b} f(\vec{a}, \vec{b})
$$

for smooth functions $f$ of compact support. Since $\Omega^{t}$ is measure preserving, this formula holds if $\Omega^{ \pm}$is replaced with $\Omega^{t}$. The desired equality then follows from Lemma 4.

\section{§ 5. Asymptotic Completeness}

Theorem 5. Let $\vec{F}$ obey (A)-(D). Then $\operatorname{ran} \Omega^{+}$and $\operatorname{ran} \Omega^{-}$differ by sets of measure 0. Equivalently (since $\Omega^{ \pm}$are isometric by theorem 4 ), for almost all $\vec{a}, \vec{b} \in R^{6}$ there is an $\vec{a}^{\prime}, \vec{b}^{\prime} \in R^{6}$ and a solution $\vec{r}(t)$ of $\ddot{\vec{r}}(t)=\vec{F}(\vec{r}(t))$ so that

$$
\begin{aligned}
& \lim _{t \rightarrow-\infty}|\vec{r}(t)-\vec{a}-\vec{b} t|+|\vec{r}(t)-\vec{b}|=0, \\
& \lim _{t \rightarrow+\infty}\left|\vec{r}(t)-\vec{a}^{\prime}-\vec{b}^{\prime} t\right|+\left|\dot{\vec{r}}(t)-\vec{b}^{\prime}\right|=0 .
\end{aligned}
$$

Proof. By a result of Hunziker and Siegel $[10,14]$, if $r(t)$ solves the equation of motion, the sets

$$
\left\{(\vec{a}, \vec{b})\left|r(0)=a, \dot{r}(0)=b, \lim _{t \rightarrow \mp \infty}\right| r(t) \mid=\infty\right\}=N_{\mp}
$$


are equal up to a set of measure zero (see Appendix 2 for an example where $N_{-} \neq N_{+}$). If we can show that when $\lim _{t \rightarrow-\infty}|r(t)|=\infty$ then $r(0)$, $\dot{r}(0) \in \operatorname{ran} \Omega^{+}$, we will be finished. We will prove this result when the energy is positive which is sufficient. By Lemma 2, it is enough to prove $\lim _{t \rightarrow+\infty} \frac{|r(t)|}{t}>0$ if $\lim _{t \rightarrow-\infty}|r(t)|=\infty$.

Let $W(t)=r(t)^{2}$ be the moment of inertia. Then $\dot{W}(\mathrm{t})=2 \dot{r} r$ $\dot{W}(t)=2 \dot{r}^{2}+2 \ddot{r} r=2 E+2 \vec{r} \cdot \vec{F}(r)-2 V(\vec{r})$ where $E=\dot{r}^{2}+V>0$, is a constant of the motion. By (B), we can find $R_{0}$ so $2 E+2 \vec{r} \cdot \vec{F}-2 V>0$ if $r>R_{0}$. Since $\varliminf_{t \rightarrow+\infty}|r(t)|=\infty$, we can find $t_{0}$ so $\left|r\left(t_{0}\right)\right|>R_{0} ; \dot{r}\left(t_{0}\right)>0$. Then $W\left(t_{0}\right)>R_{0}^{2}$ and $\dot{W}\left(t_{0}\right) \geqq 0$. It follows that $\ddot{W}>0$ for all $t>t_{0}$ and that $\dot{r}(t) \geqq \dot{r}\left(t_{0}\right)$ so that $r(t)>\left|\dot{r}\left(t_{0}\right)\right||t|$ for $t>t_{0}$.

\section{§ 6. $N$-Body Results}

We wish to note that some of our simplest results extend to the $N$-Body case without any work:

Suppose $\vec{A}, \vec{B} \in R^{3 N}$ and that $\vec{B}$ is such that if $\vec{B}=\left(\vec{b}_{1}, \ldots, \vec{b}_{N}\right)$, then $\vec{b}_{i}-\vec{b}_{j} \neq 0$ for all $i, j$. Then using the methods of Theorems 1 and 2 let us prove:

Theorem 6. Let $F_{i j}(1 \leqq i<j \leqq N)$ be given obeying (A) and (B). Let $\vec{A}, \vec{B} \in R^{3 N}$ with $\vec{b}_{i} \neq \vec{b}_{j}$ for all $i, j$. Then, there exists a solution of

$$
\frac{d r_{i}(t)}{d t}=\sum_{j \neq i} F_{i j}\left(r_{i}(t)-r_{j}(t)\right)
$$

(where $F_{i j}=-F_{i j}$ if $j>i$ ) with $\left|r_{i}(t)-a_{i}-b_{i} t\right| \rightarrow 0$ as $t \rightarrow-\infty$ and $\left|\dot{r}_{i}(t)-b_{i}\right| \rightarrow 0$. If moreover the forces obey $(\mathrm{C})$, this solution is unique.

We have not yet attempted to construct wave operators for channels other than the one where the particles are asymptotically free.

\section{§ 7. Connection with Hunziker's Approach}

Hunziker's approach involves forming $L^{2}$ of phase space and unitary maps $e^{L t}$ and $e^{L_{0} t}$ given by $\left(e^{L t} \psi\right)(z)=\psi\left(S_{-t} z\right) ;\left(e^{L_{0} t} \psi\right)=\psi\left(S_{-t}^{(o)} z\right)$ where $S_{-t}, S_{-t}^{(o)}$ are defined in $\S 4$. He proves the existance of the

$$
\lim _{t \rightarrow+\infty} e^{+L t} e^{-L_{0} t}=\Omega_{\boldsymbol{H}}^{ \pm}
$$

as a Hilbert space operator. 
When (A)-(C) holds, we proved in $\S 4$, that

$$
\lim _{t \rightarrow+\infty} S_{-t} S_{t}^{(o)}=\Omega^{ \pm}
$$

in the sense of convergence on uniform convergence on compacts. It follows that the maps $\Omega^{ \pm}$we have discussed when (A)-(C) hold are related to Hunziker's $\Omega_{H}^{ \pm}$by $\left(\Omega_{H}^{ \pm} \psi\right)(z)=\psi\left(\Omega^{ \pm} z\right)$. Thus some of Hunziker's long range results ( $\S 5$ of Hunziker's paper) follow from our theorem.

On the other hand, if one plays fast and loose with sets of measure zero, Hunziker's results imply ours. For let $M$ be a measurable set and let $\chi_{M}$ be its characteristic function. Then

$$
\begin{aligned}
{\left[\Omega_{H}^{+}\left(\chi_{M}\right)\right]^{2}(\chi) } & =\lim _{t \rightarrow-\infty}\left(\chi_{M}\right)^{2}\left(S_{-t} S_{t}^{(o)} \chi\right)=\lim _{t \rightarrow-\infty} \chi_{M}\left(S_{-t} S_{t}^{(o)} \chi\right) \\
& =\left(\Omega_{H}^{+} \chi_{M}\right)(\chi) .
\end{aligned}
$$

Thus $\Omega_{H}^{+}\left(\chi_{M}\right)=\chi_{\omega+(M)}$ for some set function $\omega^{+}$which preserves measure. In some sense $\omega^{+}$is comes from a point map. Thus, in some sense, Hunziker's theorem suggest that when only (A) and (B) hold, one has uniqueness for almost all initial data at $\infty$. This seems like a good conjecture: i.e. under conditions (A) and (B) for almost all $(\vec{a}, \vec{b}) \in R^{6}$, there is a unique solution of $\ddot{r}(t)=\mathrm{F}(r(t))$ asymptotic to $\vec{a}+\vec{b} t$ at $t=-\infty$.

\section{Appendix 1}

\section{Non Uniqueness in the Absence of Lipshitz Conditions}

We want to construct two examples of conservative systems where there are non-unique solutions of $\ddot{\vec{r}}=\vec{F}(r)$ asymptotic to $\vec{b} t$ where $|\vec{b}|=1$. Throughout, $\psi$ will represent a function which is $C^{\infty}$ on $R$ with:

$$
\psi(x)= \begin{cases}1 & \text { if } x \leqq 2 \\ \varepsilon[0,1] & \text { if } 2 \leqq x \leqq 3 \\ 0 & \text { if } x \geqq 3 .\end{cases}
$$

We use cylindrical coordinates, $(\varrho, \Phi, \mathrm{z})$.

Example 1.

$$
\begin{aligned}
V, \vec{F} & =0\left(e^{-r}\right) ; \vec{\nabla} \vec{F}=0(1) . \\
V(\varrho, \Phi, z) & =-\frac{1}{2}[1-\chi(\varrho)] Z^{2} \chi\left(z e^{\varrho}\right) \\
r_{\vec{b}}(t) & =\left(t, \Phi_{0}, b e^{-t}\right) \quad|b|<2 ; 0 \leqq \Phi_{0} \leqq 2 \pi \quad t>3 .
\end{aligned}
$$

All the functions $\vec{r}_{\vec{b}}(t)$ are asymptotic to the same free solution at $t=-\infty$. 
Example 2.

$$
\begin{aligned}
V, F & =0\left(r^{-n-2}\right) \quad\left(n>\frac{1}{2}\right) ; \vec{\nabla} \vec{F}=0\left(r^{-2}\right) \\
V(\varrho, \Phi, z) & =-(1-\chi(\varrho)) \frac{n^{2}}{2} z^{(2 n+2) / n} \chi\left(z \varrho^{n}\right) \\
\vec{r}_{\theta_{0}}(t) & =\left(t, \theta_{o}, t^{-n}\right) \quad \text { and } \quad\left(t, \theta_{o}, 0\right) \quad t>3 .
\end{aligned}
$$

These two solutions are asymptotic to the same free solution as $t \rightarrow-\infty$. Notice this example shows our $0\left(r^{-2-\varepsilon}\right)$ conditions are best possible for uniqueness.

\section{Appendix 2}

\section{An Example of Capture}

We will show here that capture, i.e. solutions of $\ddot{\vec{r}}=F(\vec{r}(t))$ with $\lim _{t \rightarrow-\infty}|\vec{r}(t)|=\infty$ while $\varlimsup_{t \rightarrow \infty}|\vec{r}(t)|<\infty$, is not a terribly pathological phenomena. For let $V$ be a smooth function going to 0 at $\infty$ as $r^{-2-\varepsilon}$ of $|\vec{r}|$ with $V\left(r_{o}\right)=V_{0}>0, V(r)<V_{0}$ for $r>r_{o} ; V\left(r_{o}\right)=0 ; V^{\prime \prime}\left(r_{o}\right) \neq 0$. Then the solution of energy $V_{0}$ and angular momentum 0 exhibits capture. For near $r_{o}, \dot{r}^{2} \sim \sqrt{E-V_{0}} \sim C\left(r-r_{o}\right)^{2}$. Thus, if $r(t) \rightarrow-\infty$ as $t \rightarrow-\infty$; as $t \rightarrow \infty,\left(r-r_{o}\right) \sim e^{-t}$.

For a general central potential, one can easily show that if capture takes place for energy, $E_{0}$ and angular momentum $l_{0}$, then

$$
V_{l_{0}}(r)=V(r)+\frac{l_{0}^{2}}{2 r^{2}}, \quad V_{l_{0}}\left(r_{o}\right)=E_{0} ; \frac{\partial V_{l_{0}}}{\partial r}\left(r_{o}\right)=0
$$

for some $r_{o}$. It then follows by Sard's theorem that this is a set of measure zero. It is amusing to notice that the Hunziker-Siegel result in this case follows from Sard's theorem!

\section{Appendix 3}

The Time Delay Formula

The usual mechanics books e.g. [15], give a simple formula for the scattering angle at a function of energy and impact parameter (or what is equivalent, angular momentum), viz:

$$
\theta=2 l \int_{r_{0}(l, E)}^{\infty} \frac{d r}{r^{2}}\left[2 E-2 V-r^{-2} l^{2}\right]^{-1 / 2}
$$

where $r$ is given by

$$
r_{o}(l, E)=\inf \left\{r \mid V(r)-\frac{l^{2}}{2 r^{2}}<E\right\} .
$$


We note here that there is a similar formula for the time delay which is an essential piece of the $S$-matrix. It is easy to derive and says:

$$
\begin{aligned}
T=2 \int_{R}^{\infty} \frac{d r}{r}\left\{\left[2 E-r^{-2} l^{2}\right]^{-1 / 2}-\left[2 E-2 V-r^{-2} l^{2}\right]^{-1 / 2}\right. \\
\quad-2 \int_{r_{o}(, E)}^{R} d r\left[2 E-2 V-r^{-2} l^{2}\right]^{-1 / 2}+2 \int_{l / \sqrt{2 E}}^{R} d r\left[2 E-r^{-2} l^{2}\right]^{-1 / 2}
\end{aligned}
$$

where $R \geqq \max \left(r_{o}, l / \sqrt{2 \mathrm{E}}\right)$.

Notice as long as $V \rightarrow 0$ at $\infty$, the integral for $\theta$ exists but for the integral for $T$ to exist, one needs more or less that $\int_{R}^{\infty} d r V(r)$ exists. This breaks down precisely at the Coulomb force as is well-known.

Acknowledgement. It is a pleasure to thank E. Nelson and M. Reed for valuable discussion.

\section{References}

1. Cook, J.: J. Math. Phys. 36, 82 (1957); Ikebe, T.: Arch. Rat. Mech. Anal. 5, 1 (1960); Jauch, J.: Helv. Phys. Acta 31, 136 (1958); Kuroda, S.: Nuovo Cimento 12, 431 (1959); Kato, T.: Math. Ann. 162, 258 (1966).

2. Amrein, W., Misra, B., Martin, Ph.: Helv. Phys. Acta, to appear; Dollard, J.: J. Math. Phys. 5, 729 (1964); Lavine, R.: J. Functional Anal. 5, 368 (1970).

3. Faddeev,L.D.: Mathematical aspects of the three-body problem in the quantum theory of scattering (Israel scientific translation, Jerusalem: Faddaev 1965); Hack, M. N.: Nuovo Cimento 13, 231 (1959); Hepp, K.: Helv. Phys. Acta 42, 425 (1969); Hunziker, W.: J. Math. Phys. 6, 6 (1965); Hunziker, W.: Mathematical theory of multiparticle quantum systems. In: Barut, Brittin (Eds.): Lectures in theoretical Physics, Vol. XA., 1968; - Jauch, J.: Helv. Phys. Acta 31, 661 (1958).

4. Chadam, J.: J. Math. Phys. 9, 386 (1968); Chadam, J.: Pacific J. Math. 31, 19 (1969); Prosser, R.: J. Math. Phys. 4, 1048 (1963).

5. Lax, P., Phillips, R.: Scattering theory. New York: Academic Press 1967; Mizohata, S.: Proc. Japan Acad. 39, 352 (1963); Morawetz, C.: Comm. Purc. Appl. Math. 14, 561 (1961).

6. Haag, R.: Phys. Rev. 112, 669 (1958); Jost, R.: The general theory of quantum fields. Providence A.M.S. 1965; Ruelle, D.: Helv. Phys. Acta 35, 147 (1962).

7. Capri, A.: J. Math. Phys. 10, 575 (1969); Schroer, B., Seiler, R., Swieca, J.: Phys. Rev. D 2, 2927 (1970); Wightman, A. S. : (in preparation); Wightman, A. S. : Partial differential equations and relativistic quantum field theory. In: Aziz (Ed.): Lectures in differential equations, Vol. II. Princeton: van Nostrand 1969.

8. Hoegh Krohn, R.: J. Math. Phys. 9, 2075 (1968); Hoegh Krohn, R.: Commun. math. Phys. To appear; Kato, Y., Mugibayshi, N.: Prog. Theor. Phys. 30, 103 (1963); Kato, Y., Mugibayshi, N.: Kobe preprint, 1970.

9. Morawetz, C.: Proc. Roy. Soc. A 306, 291 (1968); Segal, I.: Ann. Sci. Ecol. Norm. Supp. 1, 459 (1968); Strauss, W.: J. Functional Ann. 2, 409 (1968).

10. Hunziker, W.: Commun. math. Phys. 8, 282-299 (1968). 
11. Cook, J.: In: Lurcat, F. (Ed.): 1965 Cargèse lectures in theoretical physics. New York: Gordon \& Breach 1967.

12. Coddington, E. A., Levinson, N.: Theory of ordinary differential equations. New York: McGraw-Hill 1955; Goffman, C.: In: Buck, R. (Ed.): Studies in modern analysis. New York: Math. Ass. of Am. 1962.

13. Friedrichs, K.: Comm. Pure Appl. Math. 1, 361 (1948); Moller, C.: Det. Kgl. Dansk. Vid. Selsk. Math.-Fys. Medd. 23, No. 1 (1945).

14. Siegel,C.L.: Vorlesungen über Himmelsmechanik. Berlin, Göttingen, Heidelberg: Springer 1956.

15. Goldstein, H.: Classical mechanics. Reading, Mass.: Addison-Wesley 1957; Newton, R.: Scattering theory of waves and particles. New York: McGraw-Hill 1966.

16. Dunford, N., Schwartz, J.: Linear operators, Part I. New York: Interscience 1958.

17. Loomis, L., Sternberg, S.: Advanced calculus. Reading, Mass.: Addison-Wesley 1968.

Dr. Barry Simon

Departments of Mathematics and Physics

Princeton University

Princeton, New Jersey, USA 